\title{
Micro-Composites of Poly(styrene) and Nylon 3 by In-Situ Polymerization
}

\author{
Jeongwoong AnN*, Masahiro RikuKawa, Kohei SanuI, and Naoya Ogata \\ Department of Chemistry, Sophia University, 7-1 Kioi-cho, Chiyoda-ku, Tokyo 102, Japan \\ * Sam Yang R\&D Center, Sam Yang Company, 63-82 Hwaan-Dong, Yusung-Gu, Taejeon, Korea
}

(Received September 28, 1995)

\begin{abstract}
The in-situ polymerization of acrylamide in solutions of poly(styrene) (PSt) was carried out by anionic polymerization to form Nylon 3 in PSt as micro-composites in which Nylon 3 was dispersed as fine particles. Copolymers of styrene (St) and 4-vinylpyridine (4-VPy) were used as matrix polymers in order to improve a compatibility between the in-situ formed Nylon 3 and matrix polymer through hydrogen-bonding. These micro-composites showed great improvement of mechanical properties owing to reinforcement by ultra-fine particles of Nylon 3. Especially, the micro-composite derived from poly(St-co-4-VPy) showed excellent improvements of mechanical properties owing to the hydrogen bonding interactions between Nylon 3 and 4-VPy.

KEY WORDS Micro Composites / Poly(styrene) / Nylon 3 / Hydrogen Bonding /
\end{abstract}

Rigid rod-like polymers such as liquid crystalline polymers have high melting points and less solubilities in common organic solvents. ${ }^{1}$ Moreover, they are usually not compatible with commodity polymers such as poly(styrene) (PSt) and phase separations easily take place when they are blended by either solution or melt blend methods. It was previously reported that in-situ polymerization of monomers which can form rigid rodlike polymers within solutions of commodity polymers as matrix polymers lead to so-called "micro-composites," in which the rigid rod-like polymers are dispersed as ultra-fine particles so that mechanical properties of the resulting micro-composites are greatly improved in terms of tensile strength and thermal stability. Microcomposites based on liquid crystalline polyesters, ${ }^{2}$ polyaramides, ${ }^{3}$ polyglutamates ${ }^{4}$ have been already reported.

This paper deals with micro-composites of poly(styrene) and Nylon 3 which is obtained by an anionic polymerization of acrylamide. It is known ${ }^{5,6}$ that acrylamide is polymerized into Nylon 3 in the presence of basic catalysts such as potassium butoxide and Nylon 3 has a high decomposition temperature of $330^{\circ} \mathrm{C}$ and a high modulus. Therefore, it is expected that microcomposites having good mechanical properties would be obtained when acrylamide is in-situ polymerized in a solution of PSt. Interface interactions between PSt and Nylon 3 would be important to enhance the mechanical properties of micro-composites. Therefore, copolymers of styrene and 4-vinyl pyridine (4VPy) were also used as matrix polymers so that hydrogen bonding between Nylon 3 and 4VPy would enhance the compatibility between the matrix polymers and Nylon 3 .

$$
\begin{aligned}
\mathrm{CH}_{2}=\mathrm{CHCONH}_{2} \frac{t}{\mathrm{PSt} / \text { monochlorobenzene }} \\
{\left[\mathrm{CH}_{2} \mathrm{CH}_{2}-\mathrm{CONH}\right] }
\end{aligned}
$$

\section{EXPERIMENTAL}

Synthesis of Micro-Composite of PSt and Nylon 3

Poly(styrene) (PSt) was bought from the Tokyo Kasei Co. and purified by reprecipitation from $n$-hexane-eth- anol. Reagent-grade acrylamide of the Wako Chemicals was purified by recrystallization from methanol. Styrene and 4-vinylpyridine which were bought from the Wako Chemicals were purified by distillation in nitrogen atomosphere and used right after distillation.

A portion of $10 \mathrm{~g}$ of PSt having molecular weight of 200000 which was bought from the Wako Chemicals was dissolved in $100 \mathrm{ml}$ of monochlorobenzene. To this solution $4.28 \mathrm{~g}(0.0602 \mathrm{~mol})$ of acrylamide and $0.34 \mathrm{~g}$ $(0.003 \mathrm{~mol})$ of potassium tert-butoxide were added with stirring. The solution was heated at $100^{\circ} \mathrm{C}$ for $7 \mathrm{~h}$ under nitrogen atmosphere. The clear solution became milky after $3 \mathrm{~h}$ and an emulsion was obtained. After the polymerization, the entire solution was poured into excess methanol to precipitate the micro composite containing Nylon 3 . The recovered micro composite was washed with methanol repeatedly and dried under vacuum. Films were obtained by a solvent casting of monochlorobenzene solution of the recovered micro composite on a Teflon sheet.

Poly(St-co-4VPy) was obtained by a conventional radical copolymerization of St and 4VPy in bulk in the presence of azobisisobutylonitrile. After the copolymerization the copolymer was dissolved in chloroform, followed by precipitation in excess ether and collection by filtration. Molecular weight of the copolymer which was determined by solution viscosity was 180000 . The in-situ polymerization of acrylamide in poly(St-co-4VPy) solution was carried out by similar methods to the above.

\section{Characterization of Micro-Composites}

Micro-composites were characterized by a FT-IR instrument of the Shimazu FTIR-8600PC and by elemental analyses.

The in-situ formed Nylon 3 was isolated by extracting the films with benzene, followed by characterization by FT-IR and determinations of solution viscosities in sulfuric acid at a concentration of $1 \mathrm{wt} \%$ at $30^{\circ} \mathrm{C}$.

Thermal analyses of the micro-composites were carried out by the Seiko TG/DTA 200 thermal analyzer.

Fracture surfaces of the micro-composite films were observed by scanning electron microscopy (SEM) Hitachi 
S-430 to determine the dispersion of the in-situ formed Nylon 3 in PSt. The mechanical properties of the films were measured by the Shimadzu AGS-1kNA Tensilon.

\section{RESULTS AND DISCUSSION}

\section{Micro-Composites Based on PSt}

The in-situ polymerization of acrylamide in a PSt solution was carried out and the results are summarized in Table I. It is seen in Table I that yields of Nylon 3 are slightly smaller than feed ratios of acrylamide and decrease with increasing feed amount of acrylamide. The reason of less yields of Nylon 3 might be the deposition of Nylon 3 on the walls of the reactor when the feed amount of acrylamide increases. Solution viscosities of resulting Nylon 3 were in the range of 1.52 and 1.98 indicating high molecular weights of Nylon 3.

Glass transition temperatures of micro-composites increased with content of Nylon 3 in the micro-composites as shown in Figure 1. The simple increasing curve of $T_{\mathrm{g}}$ of the micro-composites in Figure 1 does not simply means apparent good compatibility between two polymers, but DSC measurements might not be sensitive to detect phase separation between two polymers, as the $T_{\mathrm{g}} \mathrm{s}$ of two plymers were close to each other. Tensile strength, tensile modulus and elongation of films of the micro-composites are shown in Figures 2, 3 , and 4 , respectively. Both tensile strength and modules increased with content of Nylon 3 in the composites, while elongation of the films slightly decreased

Table I. Synthesis of PSt/Nylon 3 micro-composities by in-situ polymerization of acrylamide (AAm) in PSt solutions

\begin{tabular}{|c|c|c|c|c|}
\hline Code No. & $\frac{\text { AAm feed }}{w t \%}$ & $\begin{array}{c}\text { Nylon } 3 \text { in } \\
\text { composites/ } \\
w^{0} \%^{a}\end{array}$ & $\begin{array}{l}\text { Yield of } \\
\text { Nylon } 3\end{array}$ & $\begin{array}{c}\eta_{\text {rel }} \text { of } \\
\text { Nylon } 3^{b}\end{array}$ \\
\hline PS-N9 & 10 & 8.8 & 87 & 1.84 \\
\hline PS-N14 & 20 & 14.4 & 68 & 1.98 \\
\hline PS-N24 & 30 & 24.3 & 75 & 1.81 \\
\hline PS-N32 & 40 & 31.8 & 70 & 1.73 \\
\hline PS-N37 & 50 & 37.3 & 59 & 1.52 \\
\hline
\end{tabular}

${ }^{\mathrm{a}}$ Content of Nylon 3 was determined by elemental analysis. ${ }^{\mathrm{b}}$ Isolation of Nylon 3 was carried out by extracting composites with benzene under refluxing conditions. Solution viscosities were determined in $98 \%$ sulfuric acid at $30^{\circ} \mathrm{C}$ at a concentration of $1 \mathrm{~g} \mathrm{dl}^{-1}$.

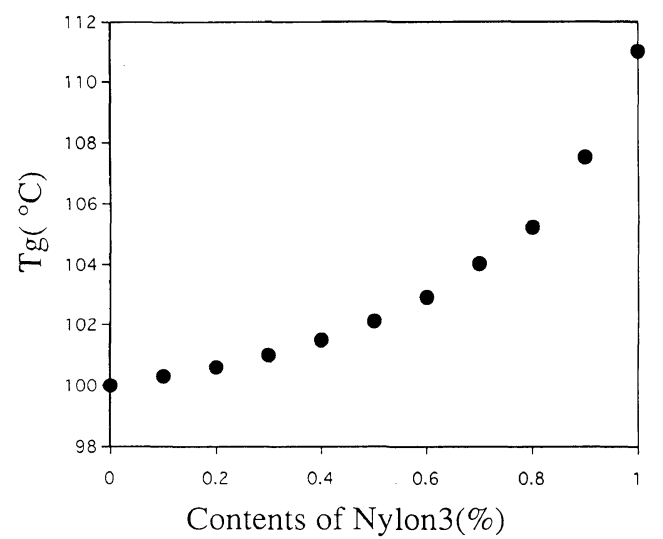

Figure 1. Glass transition temperatures of micro-composites of PSt and Nylon 3. with increasing content of Nylon 3 .

SEM pictures of the films are shown in Figure 5, which indicates that the in-situ formed Nylon 3 is dispersed as fine particles and the diameters of the fine Nylon 3 particles increased gradually from 0.1 to $0.5 \mu \mathrm{m}$ with feed ratio of acrylamide in the PSt slutions. Affinity between PSt and Nylon 3 would not be satisfactory because PSt is hydrophobic and Nylon 3 is hydrophilic and a phase separation of Nylon 3 would occur so that the domain size of Nylon 3 increases when the feed ratios of acrylamide in the PSt solution increase. Nevertheless, mechanical properties of the micro-composite films were greatly improved owing to the welldispersed state of Nylon 3 in PSt.

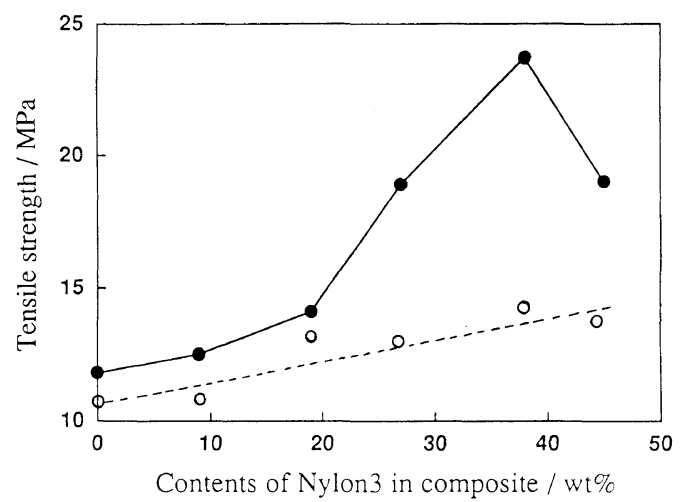

Figure 2. Tensile strength of the micro-composite films as function of Nylon 3 content: O, PSt-Nylon 3 systems; PSt/4-VPy-Nylon 3 systems.

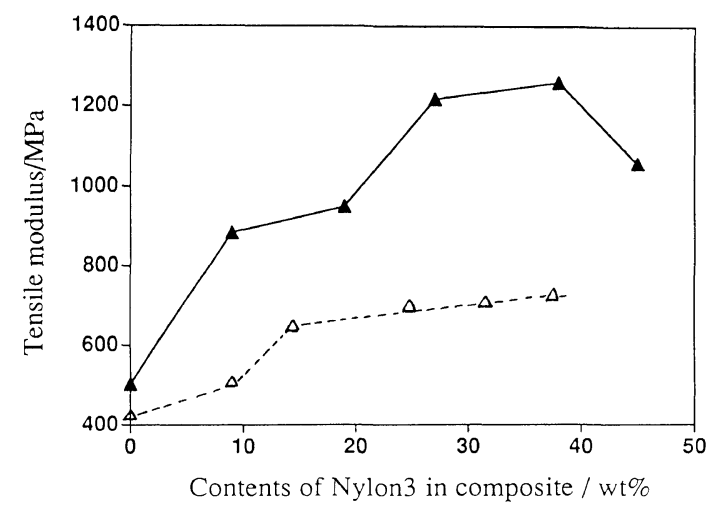

Figure 3. Tensile modulus of micro-composite films as function of Nylon 3 content: $\triangle$, PSt-Nylon 3 systems; $\mathbf{\Delta}$, PSt $/ 4-\mathrm{VPy}-\mathrm{Nylon} 3$ systems.

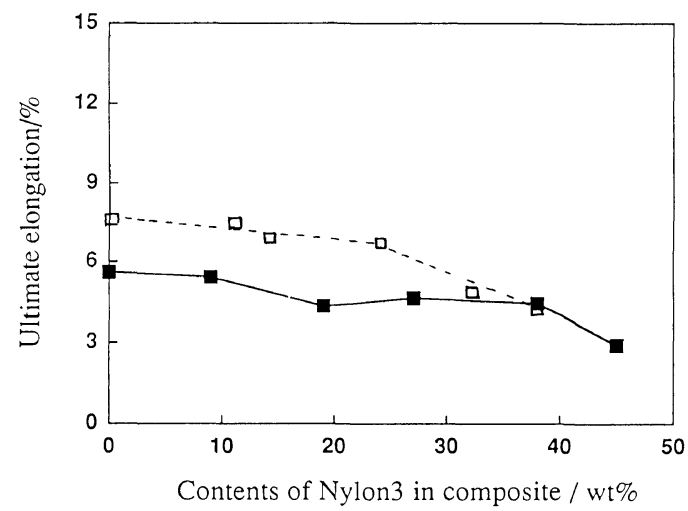

Figure 4. Elongation of the micro-composite films as function of Nylon 3 content: $\square$, PSt-Nylon 3 systems; $\square$, PSt/4-VPy-Nylon 3 systems. 


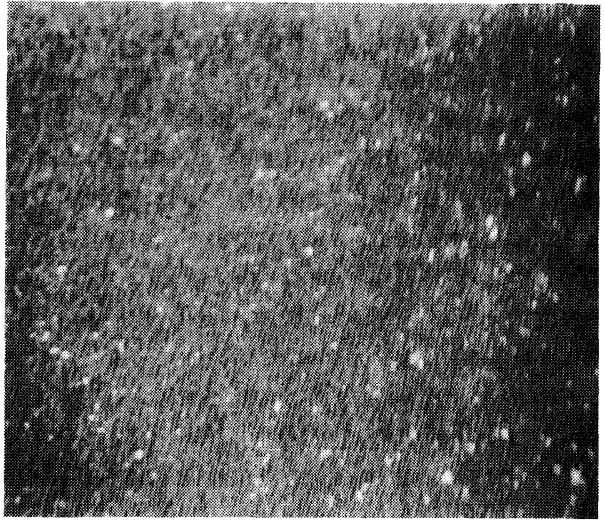

(a)

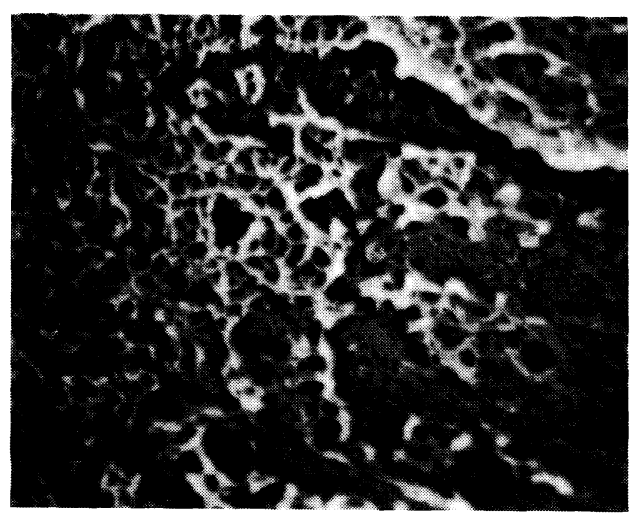

(c)

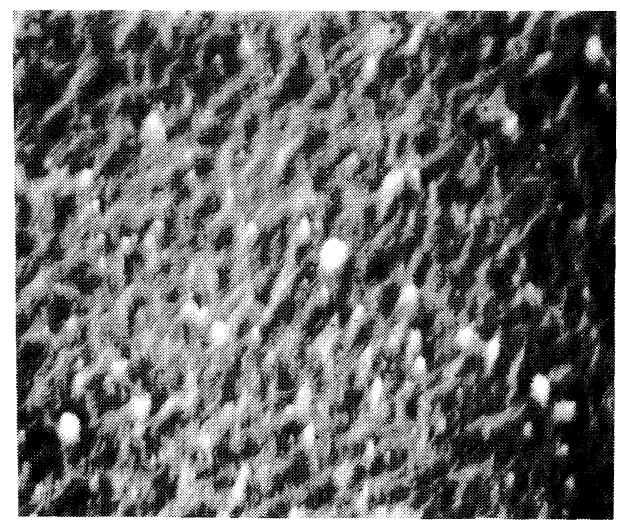

(e)

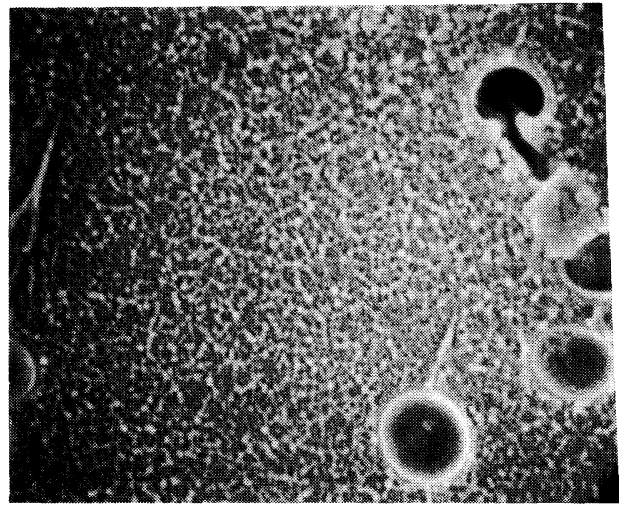

(b)
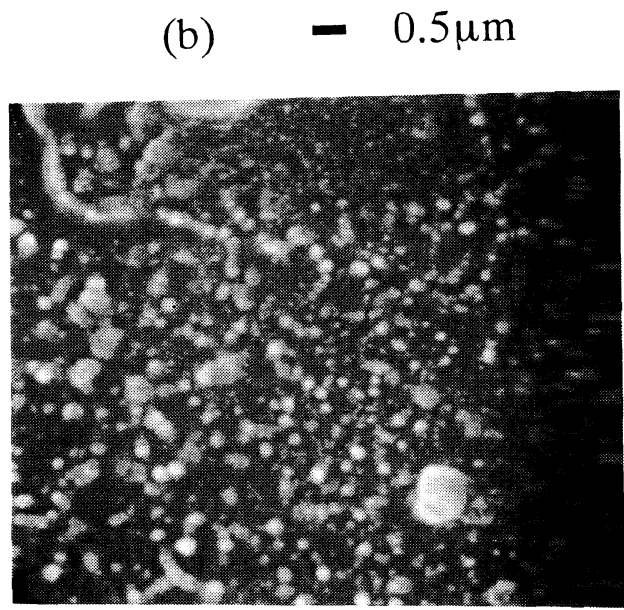

(d)

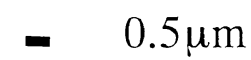

Figure 5. SEM pictures of the fractured surface of the micro-composite films of PSt/Nylon 3: (a) PS-N9; (b) PS-N14; (c) PS-N24; (d) PS-N32; (e) PS-N37.

Micro-Composites Based on the PSt-4VPy Copolymer

Enhancement of the compatibility between PSt and Nylon 3 might be achieved when an hydrophilic comonomer is incorporated into PSt. Based on this expectation 4-VPy was used as a comonomer of St, as 4VPy would form hydrogen-bond with Nylon 3 through amide linkages. A copolymer of 50/50 molar ratio of St and 4-VPy obtained by a radical copolymerization in bulk by using azobisisobutylonitrile as a radical initiator was used as the matrix polymer.

Results of the in-situ polymerization of acrylamide in the copolymer solution are summarized in Table II. It is seen in Table II that the content of Nylon 3 remained essentially the same since the feed ratios of acrylamide and yields of Nylon 3 were better than those of PSt-based micro-composites. Deposition of Nylon 3 on the reactor
Table II. Synthesis of PSt $-4 \mathrm{VP} /$ Nylon 3 micro-composities by in-situ polymerization of acrylamide (AAm) in PSt-4VP solution

\begin{tabular}{lcccc}
\hline Code No. & AAm feed & $\begin{array}{c}\text { Nylon 3 in } \\
\text { composites/ } \\
\text { wt } \%^{\mathrm{a}}\end{array}$ & $\begin{array}{c}\text { Yield of } \\
\text { Nylon 3/\% }\end{array}$ & $\begin{array}{c}\eta_{\text {rel }} \text { of } \\
\text { Nylon } 3^{\mathrm{b}}\end{array}$ \\
\cline { 3 - 4 } wt $\%$ & 9.3 & 93 & 1.66 \\
PSVP-N9 & 10 & 18.9 & 93 & 1.83 \\
PSVP-N19 & 19 & 27.2 & 87 & 1.94 \\
PSVP-N27 & 30 & 37.8 & 91 & 1.73 \\
PSVP-N38 & 40 & 45.4 & 83 & 2.08 \\
PSVP-N45 & 50 & & & \\
\hline
\end{tabular}

${ }^{a}$ Content of Nylon 3 was determined by elemental analysis. ${ }^{\mathrm{b}}$ Isola tion of Nylon 3 was carried out by extracting the composites with methanol under refluxing conditions. Solution viscosities were determined in $98 \%$ sulfuric acid at $30^{\circ} \mathrm{C}$ at a concentration of $1 \mathrm{~g} \mathrm{dl}^{-1}$. 
wall was not observed. Presumably, the better compatibility of the copolymer might enhanced the dispersion of the in-situ formed Nylon 3 in the copolymer. Solution viscosities of resulting Nylon 3 in the copolymer were better than those of PSt-based micro-composites. Presumably, the better compatibility of the copolymer with Nylon 3 enhanced the growth of Nylon 3 to higher molecular weights.

Figure 2 indicates tensile strength of the films based on PSt-4VPy/Nylon 3, micro-composites as functions of contents of Nylon 3, while Figure 3 shows tensile modulus of the films, in comparison with results of PSt-Nylon 3 micro-composites. Both Figures 2 and 3 indicate that tensile strength and modulus increase with content of Nylon 3. Tensile strength of the films reached almost

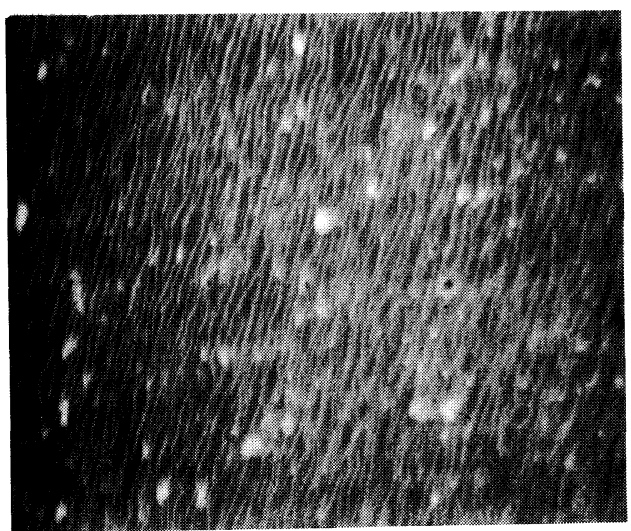

(a) $-5 \mu \mathrm{m}$

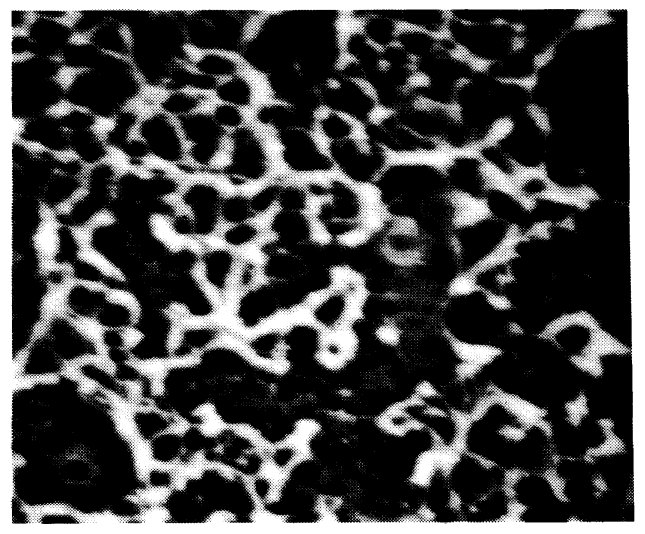

(c) $-5 \mu \mathrm{m}$

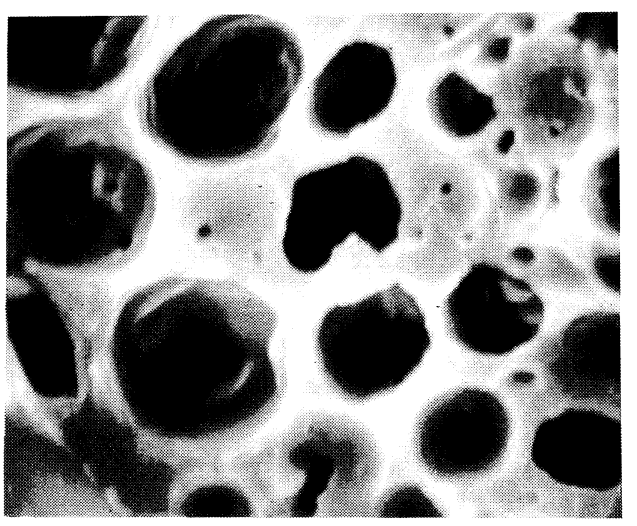

(e)

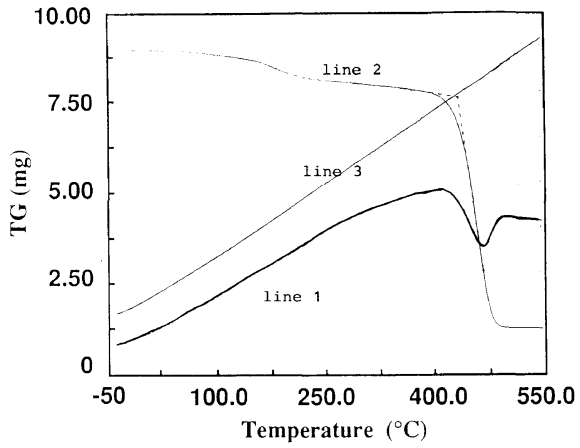

Figure 6. Thermal analysis of micro-composite film of PSt $/ 4 \mathrm{VPy}$ copolymer and Nylon 3 . Sample = PSVP-N27. Line 1, weight loss; line 2, DSC; line 3, temperature rise.

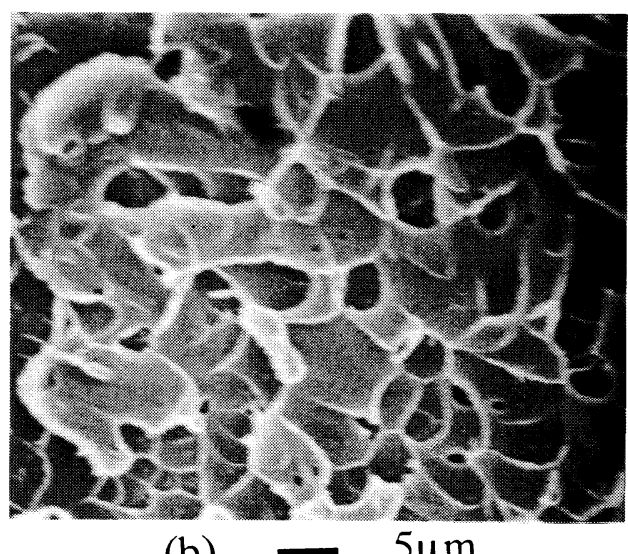

(b)

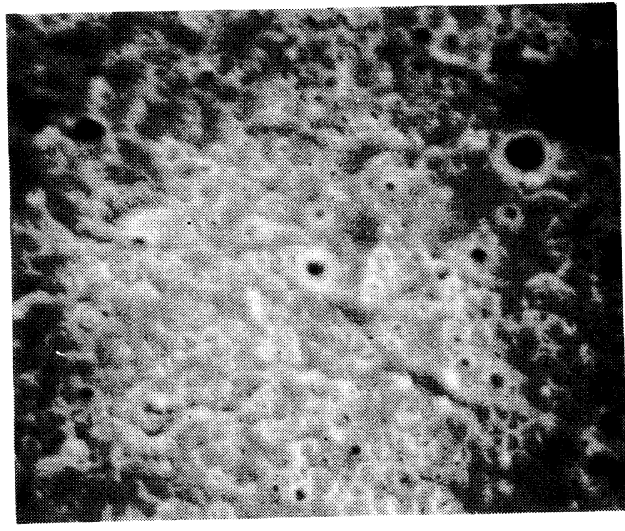

(d) $-5 \mu \mathrm{m}$

Figure 7. SEM pictures of the micro-composites of PSVP/Nylon 3: (a) PSVP-N9; (b) PSVP-N19; (c) PSVP-N27; (d) PSVP-N38; (e) PSVP-N45. 
twice that of the copolymer film, while tensile modulus increased more than twice that of the copolymer film, when Nylon 3 content was about $40 \mathrm{wt} \%$. These results were much more satisfactory than the results of PSyt- Nylon 3 micro-composites. However, both tensile strength and modulus decreased when the Nylon 3 content increased beyond $40 \mathrm{wt} \%$. Figure 4 shows elongation of micro-composite film, which decreased slightly with increasing content of Nylon 3 and the elongation of the film dropped when Nylon 3 content increased over $40 \mathrm{wt} \%$. These results suggest that a phase separation might occur when the content of Nylon 3 increased over $40 \mathrm{wt} \%$. However, improvement of the mechanical properties of the films was much better than that of the PSt-based films in tensile strength and modulus.

Thermo-gravimetric analyses of the films were carried out to study thermal decomposition behavior of the micro-composites, as shown in Figure 6. No significant change in the decomposition temperature was observed. No glass transition temperatures of the micro-composites were detected. Perhaps, better compatibility between the copolymer and Nylon 3 might prevent the mobility of the copolymer so that there is no clear glass transition temperature.

SEM pictures of the micro-composites of PSVP/Nylon 3 are shown in Figure 7 which indicate that the domain size of the in-situ formed Nylon 3 range 0.1 to $1 \mu \mathrm{m}$ and no clear interface between the copolymer and Nylon 3 was observed in comparison with the micro-composites from PSt/Nylon 3. Thus, the compatibility between the matrix polymer and Nylon 3 was improved, possibly by hydrogen bonding between pyridine and amide linkages. The domain size of Nylon 3 rapidly increased to $1-30 \mu \mathrm{m}$ when Nylon 3 content was more than $40 \mathrm{wt} \%$ and a phase separation between the copolymer and Nylon 3 was observed. Thus, the mechanical properties of the micro-composite are best at $40 \mathrm{wt} \%$ Nylon 3, owing to the phase separation. However, the mechanical properties of the micro-composite based on the copolymer of St and 4VPy were greatly improved in comparison with PSt-based micro-composites, possibly owing to the better compatibility between Nylon 3 and 4VPy units in the copolymer.

Acknowledgments. This work was partially supported by a Grant-in-Aid for Scientific Research (No. 05453146) from the Ministry of Education, Science, and Culture of Japan and the Sam Yang Company in Korea and the authors express many thanks for these supports.

\section{REFERENCES}

1. R. A. Gaudiana, R. A. Minns, R. Sinta, N. Weeks, and H. G. Rogers, Prog. Polym. Sci., 14, 47 (1989).

2. N. Ogata, K. Sanui, and H. Itaya, Polym. J., 22, 85 (1990).

3. E. Depeaute, M. Watanabe, K. Sanui, and N. Ogata, Chem. Mater., 4, 1122 (1992).

4. Y. Ayaki, M. Rikukawa, M. Watanabe, K. Sanui, and N. Ogata, Polm. J., 26, 325 (1994).

5. D. S. Breslow, G. E. Hulse, and A. S. Matlack, J. Am. Chem. Soc., 79, 3760 (1957).

6. N. Ogata, Bull. Chem. Soc. Jpn., 33, 906 (1960). 